\title{
Christopher Michael BERARD, Arthurianism in Early Plantagenet England: from Henry II to Edward I
}

\section{Nolwena Monnier}

\section{(2) OpenEdition}

\section{Journals}

Édition électronique

URL : https://journals.openedition.org/ccm/6768

DOI : $10.4000 / \mathrm{ccm} .6768$

ISSN : 2119-1026

\section{Éditeur}

Centre d'études supérieures de civilisation médiévale/Université de Poitiers

\section{Édition imprimée}

Date de publication : 1 janvier 2021

Pagination : 64-65

ISBN : 978-2-490783-08-3

ISSN : 0007-9731

Référence électronique

Nolwena Monnier, «Christopher Michael BERARD, Arthurianism in Early Plantagenet England: from Henry II to Edward I », Cahiers de civilisation médiévale [En ligne], 253 | 2021, mis en ligne le 01 mars 2021, consulté le 24 novembre 2022. URL : http://journals.openedition.org/ccm/6768 ; DOI : https://doi.org/ $10.4000 / \mathrm{ccm} .6768$

\section{(c)}

Creative Commons - Attribution - Pas d'Utilisation Commerciale - Pas de Modification 4.0 International - CC BY-NC-ND 4.0

https://creativecommons.org/licenses/by-nc-nd/4.0/ 
Christopher Michael Berard, Arthurianism in Early Plantagenet England: from Henry II to Edward I, Woodbridge, The Boydell Press (Arthurian Studies, 88), 2019.

Docteur de l'Université de Toronto, Christopher Berard enseigne actuellement au Providence College. Dès son introduction, l'a. expose très clairement la méthodologie qu'il a choisi d'adopter et insiste tout spécialement sur les sources qu'il a utilisées pour son analyse. À côté des chroniques et des romances arthuriennes - sources classiques lorsque l'on s'intéresse au personnage d'Arthur et à son imaginaire - l'a. a choisi de s'attarder sur d'autres types de sources tels les livres de lois, les traités politiques ou encore des écrits religieux. Il précise que ces sources sont en anglais mais également en latin et en anglo-normand, élément inédit selon lui. L'aspect géographique de l'analyse est également souligné. L'a. annonce ensuite son plan. Après avoir examiné le rôle d'Arthur auprès des premiers Plantagenêt, il souligne le recul pris par Richard Cœur de Lion par rapport à ce personnage et les relations entretenues par Jean sans Terre avec ce dernier. C. Berard poursuit son analyse et nous indique que la situation bascule à nouveau avec Richard, le jeune frère d'Henri III, fervent admirateur du souverain celte. Enfin, l'a. s'arrête sur le règne d'Édouard I qui reprend le contrôle sur 1'histoire d'Arthur. Cette analyse chronologique s'arrête à Édouard I. Ce choix peut paraître arbitraire au lecteur mais l'a. nous explique avoir déterminé cette date en raison du rattachement du Pays de Galles à l'Angleterre qui marque, pour lui, une étape dans les relations entre la couronne anglaise et l'imaginaire arthurien. Enfin, il expose également son but : analyser la réception au Moyen Âge du folklore arthurien. L'intérêt de l'ouvrage ne réside pas réellement sur ce point mais plutôt sur l'analyse que nous propose l'a. des lieux arthuriens et de leur influence sur le pouvoir politique de cette période. Un arbre généalogique renseigne le lecteur sur la dynastie Plantagenêt dans son ensemble.

Le premier chapitre commence par l'œuvre de Layamon. L'a. reprend alors les éléments classiques des analyses concernant l'imaginaire arthurien. Alors que le comparatif entre Arthur et Charlemagne est un classique, celui entre David, pour le peuple juif, et Arthur, pour les Celtes, est plus novateur. L'a. aborde les premières œuvres mentionnant Arthur, notamment celles de Guillaume de Malmesbury, la Gesta Regum Anglorum, et celles de Geoffroy de Monmouth en s'attardant non seulement sur l'Historia Regum Britanniae mais également sur les Prophetia Merlini et la Vita Merlini. C. Berard analyse ensuite le règne d'Henri II Plantagenêt. Les informations purement historiques sur l'accession au pouvoir du comte d'Anjou et sa légitimité contestable sur le trône d'Angleterre se succèdent. L'a. insiste ensuite sur l'analyse de l'ouvrage d'Étienne de Rouen, la Draco Normannicus et tout particulièrement sur le supposé échange épistolaire entre Arthur et Henri II. L'ouvrage a le mérite de citer abondamment les textes primaires. Il croise les sources, notamment les sources historiques et les sources purement littéraires, tels les ouvrages de Chrétien de Troyes, mais également les sources arthuriennes et les sources non arthuriennes tels les ouvrages de Pierre de Blois ou Gautier de Châtillon.

Le deuxième chapitre se consacre à Richard Cœur de Lion et s'attarde tout spécialement sur la découverte de la sépulture d'Arthur à l'abbaye de Glastonbury et sur Arthur de Bretagne, héritier malheureux du tout nouveau souverain. Des passages des ouvrages de Giraud de Barri et de Raoul de Coggeshall sont cités et l'a. développe tout particulièrement les conséquences politico-religieuses de l'accession au pouvoir de Richard Cœur de Lion et les changements qui ont pu intervenir durant cette période de transition. L'a. évoque également la rivalité entre deux lieux de sépulture : Le Mont Etna en Sicile et le Pays de Galles. Il évoque ensuite l'«espoir Breton», la croyance en le retour d'Arthur incarné par Arthur de Bretagne, fils de Geoffrey et Constance de Bretagne, neveu de Richard Coeur de Lion que ce dernier ne tarda pas à désigner comme son héritier à la couronne d'Angleterre. Une nouvelle fois, les enjeux politiques présents à cette occasion sont soulignés par l'a.

Dans le chapitre suivant, Jean sans Terre entre en scène. L'a. explique dans un premier temps l'«antiarthurianisme» du nouveau souverain dont le point d'orgue est sans nul doute la disparition suspecte du jeune Arthur de Bretagne, potentiel rival au trône. Une nouvelle fois, les problématiques politiques sont mises en avant et notamment le lien avec la cour de France où le jeune Arthur résida quelque temps. L'a. aborde également la rivalité avec la cour de Castille dont la reine, Éleanor, n'était autre qu'une des sœurs de Jean sans Terre. Pourtant, même si Jean rejette clairement Arthur, Les deux souverains sont néanmoins comparés dans l'Histoire des Ducs de Normandie et des Rois d'Angleterre. Utilisé comme outil de propagande par les barons anglais au moment de la signature de la Magna Carta Arthur est un personnage incontournable du règne de Jean alors même que ce dernier tenta de s'en détacher. 
Le chapitre 4 nous invite à poursuivre la chronologie des rois anglais avec Henri III. Fils de Jean sans Terre, ce dernier ne se révéla pas particulièrement arthurien. C. Berard nous indique en effet que ce souverain se tourna vers son oncle, Richard Cœur de Lion, le croisé, comme modèle. C'est Richard de Cornouailles, jeune frère d'Henri III, qui endosse le rôle d'admirateur d'Arthur : en 1233, il se porte d'ailleurs acquéreur du château de Tintagel - lieu supposé de la naissance d'Arthur - qu'il rénove et agrandit. Cette période de l'histoire voit aussi l'exportation de l'arthurianisme dans le reste de l'Europe, notamment en Scandinavie et dans le Saint-Empire romain germanique. Mais Arthur se révèle également être très populaire au Pays de Galles, ce qui apparaît beaucoup plus problématique à la couronne anglaise. Henri III a été propulsé dès sa montée sur le trône dans cet imaginaire arthurien et même s'il n'y adhérait pas particulièrement, C. Bérard nous explique que le souverain anglais est apparu, de son vivant, dans plusieurs romances arthuriennes en prose. Ces romances reliaient clairement la dynastie Plantagenêt au roi Arthur comme dans le cycle de la vulgate/Lancelot Graal par ex. Les liens entre Arthur et le Pays de Galles et son rôle dans la seconde guerre des barons sont également soulignés par l'a.

Le dernier chapitre est consacré à Édouard I, fils d'Henri III. L'a. suppose que le souverain a été accoutumé à la légende arthurienne par sa femme et son beau-frère castillans. Il revient également sur la croyance selon laquelle Henri III aurait donné la couronne d'Angleterre à Éleanor, sœur d'Arthur de Bretagne. Cette dernière la lui aurait alors rendue pour qu'il la donne à son fils Édouard, tissant ainsi un lien direct entre Arthur de Bretagne, l'héritier de Richard Cœur de Lion, et Édouard, nouveau souverain d'Angleterre. C'est seulement dans ce dernier chapitre que l'a. établit clairement la distinction entre l'Arthur de l'Histoire et l'Arthur des romances. Huitième croisade? Expansion vers le
Pays de Galles? Arthur est de ces deux événements marquants du règne d'Édouard. C. Berard souligne que l'autre acte significatif du règne d'Édouard est la translation, en 1278, des ossements d'Arthur à l'abbaye de Glastonbury, le souverain anglais établissant clairement le lien qui l'unissait au roi celte.

La mort de Llywelyn et de Dafydd, derniers souverains gallois, ouvre le chemin pour Édouard. Mais, comme le souligne l'a., les ambitions territoriales de celui-ci vont également vers le nord. La mort de Margareth de Norvège, héritière d'Écosse rend la situation délicate puisqu'elle était fiancée au fils ainé du souverain anglais. Une alliance franco-écossaise se profile alors, situation inenvisageable pour Édouard. Arthur refait alors son apparition dans les écrits. L'a. nous explique également que plusieurs tables rondes eurent lieu, notamment à Kenilworth et à Falkirk, signant matériellement le rattachement de la couronne anglaise à l'imaginaire arthurien.

Une bibliographie clôture l'ouvrage. Malheureusement, beaucoup de références sont assez anciennes (même si incontournables sur le sujet), et exclusivement anglophones ce qui prive le travail proposé d'un certain nombre d'analyses plus récentes et non anglophones.

Cet ouvrage, au-delà de synthétiser l'appropriation, ou non, du personnage d'Arthur et de son univers par la cour Plantagenêt, retrace les tendances de chaque chroniqueur en faveur ou non de la couronne anglaise. Le nombre de sources analysées est impressionnant et c'est son principal mérite puisque ses conclusions restent classiques sur les éléments analysés. Particulièrement bien contextualisé, cet ouvrage est un beau panorama de la politique des Plantagenêt, que le lecteur cherche à en savoir plus, ou non, sur les liens entre la couronne anglaise et le roi celte.

Nolwena MonNiER

ÉA 2557 - CÉMA Université Paris Sorbonne 